\title{
Autonomous oscillatory shape change of DEA induced by the charge-discharge process under a constant voltage
}

\author{
Hirohisa Tamagawa*, Masaki Kakihana and Minoru Sasaki \\ Department of Mechanical Engineering \\ Faculty of Engineering, Gifu University, 1-1 Yanagido, Gifu, Gifu 501-1193, Japan \\ *tmgwhrhs@gifu-u.ac.jp
}

Received 31 October 2016; Revised 28 December 2016; Accepted 29 December 2016; Published 31 January 2017

\begin{abstract}
Despite the promising characteristics of Dielectric Elastomel Actuator (DEA) as a practical soft actuator, the need of high voltage for its operation prevents the successful fabrication of a practical DEA, that is, the high voltage generation takes a bulky and costly power supply. Induction of complex shape change motion of DEA such as oscillatory shape change takes even a more bulky and costly multipurpose power supply. It is a serious practical issue to be overcome. In our latest study, however, we could build a simple DEA system which exhibited a relatively complex and autonomous oscillatory shape change merely under a constant voltage, though the voltage needed was high. This successful outcome must broaden the potential usefulness of DEA as a practical soft actuator.
\end{abstract}

Keywords: DEA; oscillation; shape change; charge-discharge.

\section{Introduction}

Soft actuators have attracted broad attention owing to their quite uncommon characteristics for an actuator. Low mass, soft motion, low consumption energy are some of such characteristics commonly possessed by the various soft actuators such as hydrogel, Ionic Polymer Metal Composite (IPMC), conducting polymer and etc. ${ }^{1-4}$ Among these various kinds of soft actuators, Dielectric Elastomer Actuator (DEA) is a bit unusual one in that, for instance, it does not need a solvent for its activation unlike other soft actuators, the current in need for the DEA operation is quite low for an electroactive polymer actuator. ${ }^{3,5,6}$ These characteristics are quite favorable properties for achieving a practical soft actuator. On the other hand, a quite high voltage on the order of a few $\mathrm{kV}$ is to be imposed on the DEA for its operation. ${ }^{3,5,6}$ From the view of safety issue, the use of such a high voltage should be avoided especially when the DEA is used in our daily life. So, the high voltage use is a significant drawback for the DEA practical use.

Basic structure of DEA is the same as a capacitor: a dielectric elastomer is sandwiched between two flexible electrodes. The DEA shape change is caused by the Maxwell stress. ${ }^{3,5,6}$ Therefore, the DEA shape change is controllable by the control of voltage imposed. However, the high voltage control is not an easy task. Hence, we cannot control the DEA shape so precisely at our will. For instance, the induction of high frequency shape change of DEA, which is in a certain situation definitely required for the practical DEA, takes the high frequency alternate high voltage. But the generation of high frequency alternate high voltage is not so easily achieved, and even though it is achievable, some costly and relatively bulky apparatuses are in need. It is one of the major problems hobbling the practical DEA fabrication. Of course, various types of simple and/or complex shape changes other than the oscillatory shape change are required for the practical DEA as well. Among these various types of shape changes, we tried to achieve the oscillatory shape changeable DEA as a first attempt, which is autonomously induced under a constant voltage instead of an alternate voltage. The outcome was basically successful, and it moves the DEA study forward to some extent to the ultimate goal of achieving a practical DEA.

\section{Maxwell Stress and DEA Shape Change}

Structure of DEA is illustrated in Fig. 1(a). Maxwell stress, which is generated by the charge induced in the flexible electrodes upon the high voltage impose, results in the DEA shape change, thinning and in-plane extension as illustrated in Fig. 1(b) ${ }^{3,5,6}$ Discharge restores the shape of DEA (see Fig. 1(b) again). With this relationship between the DEA shape and charge-discharge process in mind, we designed an oscillatory shape changeable DEA under a constant imposed voltage.

\section{Experiment}

\subsection{Fabrication of DEA}

We attempted to fabricate a DEA which exhibits the autonomous oscillatory shape change under a constant voltage, and

This is an Open Access article published by World Scientific Publishing Company. It is distributed under the terms of the Creative Commons Attribution 4.0 (CC-BY) License. Further distribution of this work is permitted, provided the original work is properly cited. 


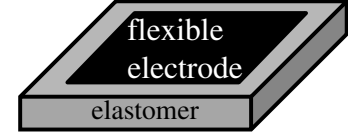

bird's eye view

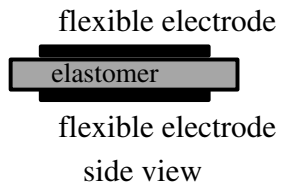

(a)

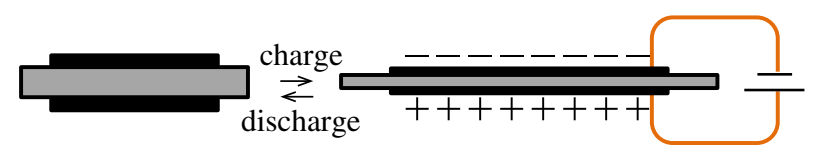

(b)

Fig. 1. (a) Basic DEA structure. (b) DEA shape change by Maxwell stress.

not under an alternate voltage. ${ }^{7}$ We used an elastomer of $1 \mathrm{~mm}$-thick VHB tape (3M, Japan). ${ }^{8}$ A $3 \mathrm{~cm}$-diameter circular VHB tape was stretched three-fold and fixed on the circular acrylic frame (inner diameter $=7 \mathrm{~cm}$, outer diameter $=9 \mathrm{~cm}$ ) as illustrated in Fig. 2(a). ${ }^{7}$ Flexible electrodes indicated by A, $\mathrm{B}, \mathrm{C}$ and $\mathrm{D}$ and the electric paths connecting those individual flexible electrodes and the positive or negative terminal were formed by painting carbon grease on the VHB tape surfaces as illustrated in Fig. 2(b). ${ }^{7}$ Then, two metal plates (aluminum

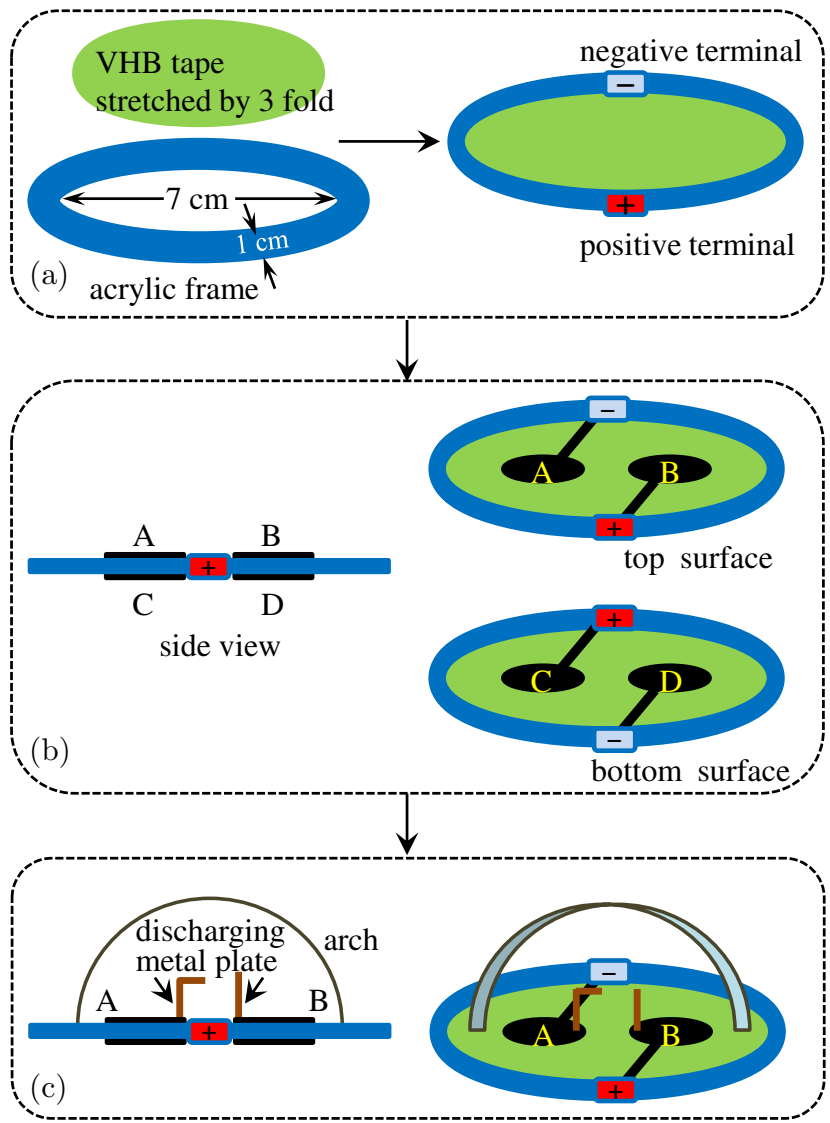

Fig. 2. Fabrication procedure of DEA (O-DEA).

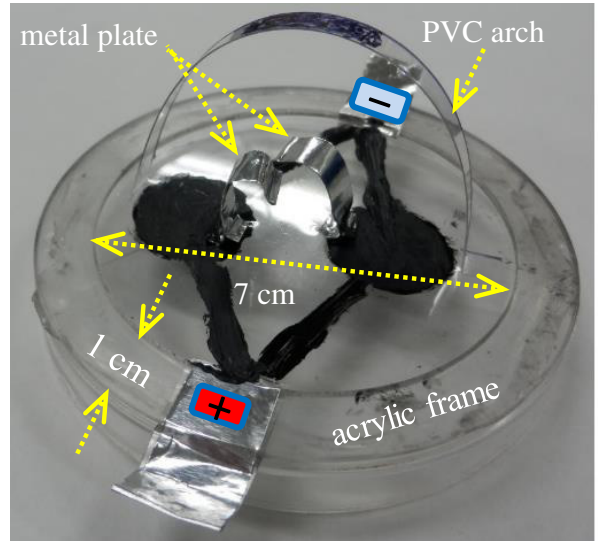

(a)

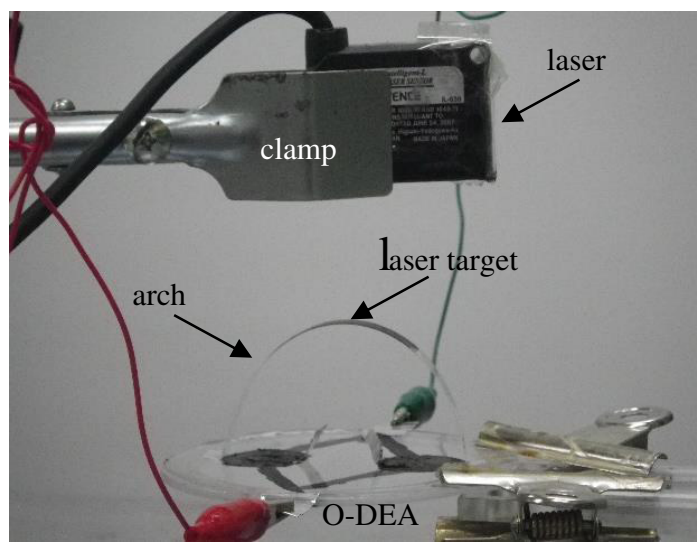

(b)

Fig. 3. (a) O-DEA. (b) Experimental setup + is connected to the positive terminal of power supply, while - is connected to the negative terminal.

adhesive tape) were placed on the electrodes A and B as illustrated in Fig. 2(c), both would serve as a discharging electrode. A flexible arch made of polyvinylchloride plate $(10 \mathrm{~cm} \times 0.5 \mathrm{~cm} \times 0.2 \mathrm{~mm}$, Young's modulus $=4.1 \mathrm{GPa})$ was attached on the top surface of VHB tape as illustrated in Fig. 2(c). The photo of DEA fabricated is shown in Fig. 3(a), and hereafter we call this DEA "O-DEA (Oscillatory DEA)".

\subsection{Measurement}

Figure 4(a) shows the O-DEA in the OFF state. Imposing constant voltage on it induces the charge in the individual electrodes: Electrodes B and C are positively charged, while electrodes $\mathrm{A}$ and D are negatively charged. Due to the Maxwell stress induced between electrodes $\mathrm{A}$ and $\mathrm{C}$, these two electrodes attract each other and consequently, they extend in-plane direction, and so do the electrodes B and D. Such deformation of electrodes moves the position of piers of arch outward, resulting in the height change of arch as illustrated in Fig. 4(b). Simultaneously, the gap between the 


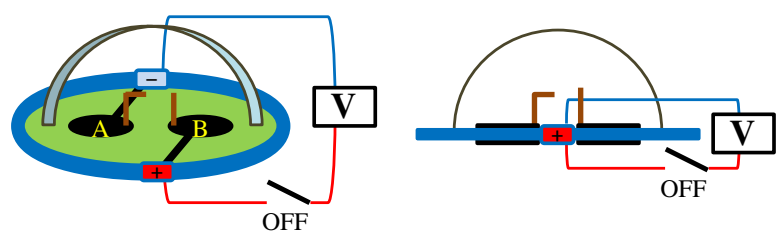

(a)

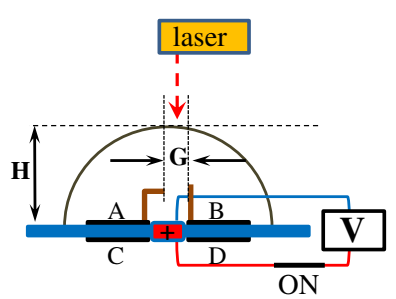

discharged state

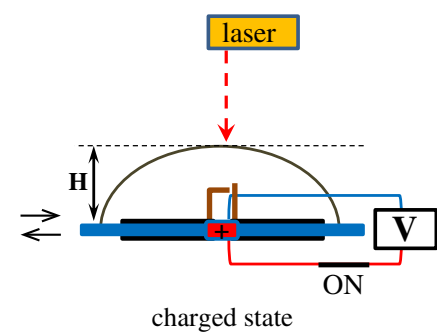

(b)
Fig. 4. (a) O-DEA in the OFF state. (b) O-DEA in the ON state.

two discharging metal plates narrows, where the gap, G, is defined as in the illustration in Fig. 4(b). Once the state of quite narrow $\mathrm{G}$ is achieved, the discharging is abruptly induced through the discharging metals. It leads to the restoration of the shape of all electrodes due to the loss of charge stored in the individual flexible electrodes (A, B, C and D), and it leads to the restoration of arch shape. Hence, the change of arch height indicated by $\mathrm{H}$ in Fig. 4(b) is induced by this process, and the height change reflects the shape change of VHB tape part of O-DEA quite well.

Since it is quite difficult to precisely measure the in-plane shape change of DEA, we made measurement of the height change of arch, $\mathrm{H}$, as a function of time using the laser displacement sensor, where the actual experimental setup photo is shown in Fig. 3(b). Simultaneously, the time dependence of current induced was measured, too.

\section{Results and Discussion}

\subsection{VHB tape-based DEA}

A measurement described in Sec. 3.2 was performed. The procedure is described again in order to have the results well understood. O-DEA was connected to the power supply as illustrated in Fig. 4(a), and actual photo is shown in Fig. 3(b). After turning on the switch as illustrated in Fig. 4(b), the voltage imposed was slowly raised by manually rotating the voltage dial of power supply. We found that once the voltage reached $2.8 \mathrm{kV}$, the charge-discharge process described in Sec. 3.2 was autonomously induced repeatedly, and the voltage imposed was maintained at $2.8 \mathrm{kV}$ from this moment on.

Time dependence of change of arch height and current induced were measured, where the onset time of this autonomous charge-discharge process is defined time $t=0 \mathrm{~s}$.

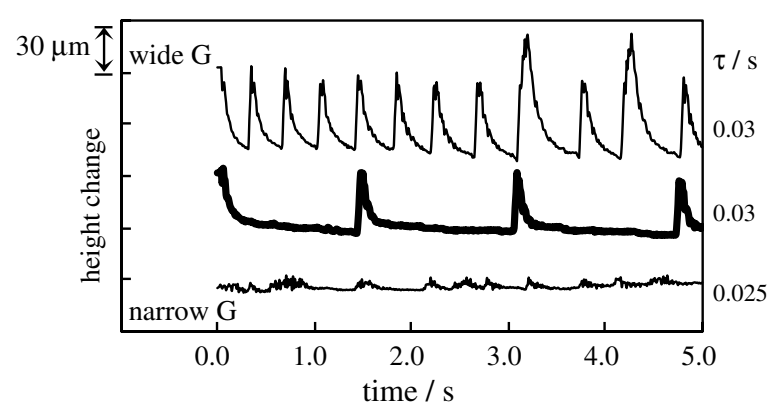

(a)

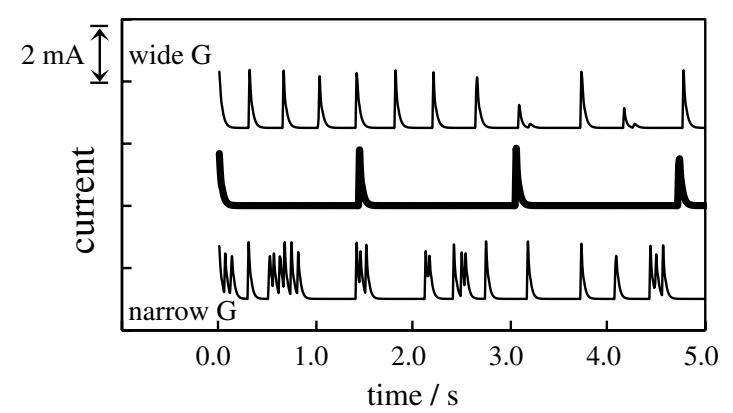

(b)

Fig. 5. (a) Height change $\mathrm{H}$ versus time, characteristic time, $\tau$, of individual curves are shown on the right of this diagram. (b) Current induced versus time. Thick data curves in (a) and (b) were obtained by the impose of $2.8 \mathrm{kV}$ on the O-DEA.

Thick data curves in Figs. 5(a) and 5(b) were the experimental results. Without the use of alternate voltage, autonomous oscillatory shape change of O-DEA was actually achieved. Its shape change frequency was about $0.8 \mathrm{~Hz}$, and it synchronized with the current behavior. The mechanism of this phenomenon is explicable by the concept described in Sec. 3.2.

The arch height change is heavily dependent on $G$, and it means that $G$ is one of the fundamental factors ruling the degree of DEA shape change. Prior to imposing the voltage on the O-DEA, the initial G at OFF state is set wider. It could raise the voltage, at which the autonomous charge-discharge process occurs, beyond $2.8 \mathrm{kV}$. It must consequently result in the larger in-plane extension of electrodes A, B, C and D, causing the large change of arch height. As a matter of fact, we could experimentally confirm it as represented by the data curve denoted by "wide G" in Fig. 5(a), and of course, the shape change behavior synchronized with the current behavior represented by the data curve is denoted by "wide G" in Fig. 5(b). Similarly, setting the initial G at OFF state narrower must take the voltage less than $2.8 \mathrm{kV}$ for the induction of autonomous oscillatory shape change of DEA and it must result in the small change of arch height synchronizing with the current behavior. Indeed, we experimentally confirmed it as shown by the data curves denoted by "narrow G" in Figs. 5(a) and 5(b). The characteristic time, $\tau$, of the 
three data curves in Fig. 5(a) was calculated and shown in Fig. 5(a).

The data curve of current, $I(t)$, represented by the thick line in Fig. 5(b) was numerically converted into the charge, $Q(t)$, by Eq. (1).

$$
Q(t)=\int_{0}^{t} I(\tau) d \tau .
$$

$Q(t)$ is shown in Fig. 6 by the dotted line along with the data curve of height change represented by the solid line which is the same data curve of the height change represented by the thick line in Fig. 5(a). As clearly seen in Fig. 6, the arch height changed between $\sim 0 \mu \mathrm{m}$ and $\sim-33 \mu \mathrm{m}$ periodically. Abrupt change of height observed at the moments indicated by the dashed arrows in Fig. 6 requires almost perfectly the same quantity of charge stored, $Q_{H}$, in the electrodes of A, B, $\mathrm{C}$ and $\mathrm{D}$ as shown in Fig. 6. Similarly, we could confirm that the height change requires the constant quantity of charge stored for the O-DEA with the narrow G shown in Fig. 5, and it is also the case with the O-DEA with the wide G shown in Fig. 5. These are quite amazing characteristics, since the DEA consists of primarily polymeric materials and the components of polymeric materials are randomly oriented polymer chains. Contrary to our intuition, the O-DEA consisting of such randomly oriented polymer chains exhibited quantitatively well-regulated shape change behavior. This fascinating characteristic will be investigated in the next section.

Judging from what is so far described, in the DEA could be used as a functionally superior actuator near future, which can exhibit a complex but precise deformation such as highprecision oscillation. Probably, the people who are willing to use the practical DEA have a strong interest in the primary factors ruling the oscillation frequency of DEA. Our research results indicate that at least the following factors rule the oscillation frequency: (1) dielectric constant of elastomer, (2) Young's modulus of elastomer, (3) static capacitance of DEA, (4) the magnitude of voltage imposed, (5) quantity of charge stored in the DEA, (6) discharging electrode gap G, (7) diameter of individual flexible electrodes A, B, C and D, (8) mutual gap among the flexible electrodes A, B, C and D, etc. Since the DEA deformation depends on its mechanical and

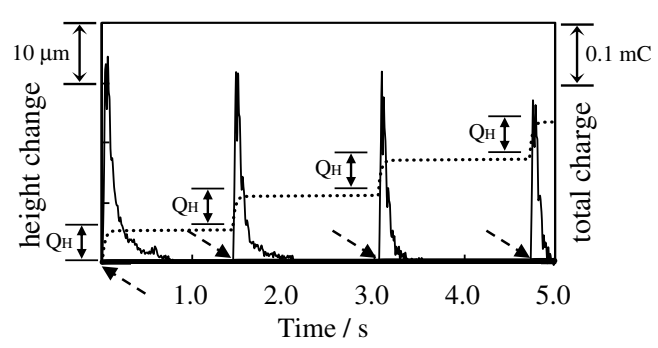

Fig. 6. Time dependence of height change of O-DEA (solid line) and of the total charge imposed on the O-DEA (dotted line). The solid line data curve is the same data curve of thick line in Fig. 5(a). electrical properties, there are a number of factors ruling the oscillation frequency. In order to use the DEA in a practical way, it is necessary to theorize the DEA deformation behavior in response to the external electrical stimulations by taking into consideration the DEA mechanical and electrical properties. That is our immediate future task.

\subsection{Silkron-based DEA}

Since the active component of O-DEA is a VHB tape consisting of randomly oriented polymer chains, we did not anticipate the quantitatively well-regulated shape change O-DEA under the external electrical stimulation. Contrary to our anticipation, the O-DEA actually exhibited the electrically well-regulated shape change as described in Sec. 4.1. This unexpected characteristic is quite fascinating even from the standpoint of electroactive polymer study. A number of researchers have been involved in the investigation of various kinds of electroactive polymers for the purpose of fabricating a practical electrically driven soft actuator. Usually, the electroactive polymer shape change is not so well electrically controllable. Achieving the precise shape change control of electroactive polymer is one of challenging tasks in the electroactive polymer research field. For example, one of the electroactive polymers called IPMC (Ionic Polymer-Metal Composite) is a bending mode electroactive polymer, and two of the authors of this paper (H.T. and M.S.) have for long years investigated IPMC. ${ }^{9-11}$ So far, they have achieved the electrical bending control only under the limited condition. Hence, DEA exhibits unusual performance as a precisely shape-controllable electroactive polymer.

We fabricated another DEA in order to see if the DEA has precisely controllable deformation commonly. If it is actually possible, we can expect the great potential usefulness of DEA as an actuator. We fabricated a Silkron-based DEA by the fabrication procedure illustrated in Fig. 7. The fabrication procedure is basically same as that of O-DEA illustrated in Fig. 2, but no discharging metals were used. This Silkronbased DEA, whose structure is shown in Fig. 7(c), is hereafter called S-DEA, where Silkron is a polyurethane sheet produced by Okura Industrial CO., Ltd. (Kagawa, Japan) and $40 \mu$ m-thick Silkron was used for S-DEA. Since Silkron is not largely stretchable as VHB tape, the Silkron was only slightly stretched as in Fig. 7(a) for fabricating an S-DEA.

S-DEA underwent the alternate voltage application of constant $1.5 \mathrm{kV}$ and $0 \mathrm{kV}$ repeatedly and the change of arch height was measured under this voltage application process. The arch height changed alternately between $0 \mu \mathrm{m}$ (under $0 \mathrm{kV}$ ) and $13 \mu \mathrm{m}$ (under $1.5 \mathrm{kV}$ ) in response to the voltage application. For the induction of $13 \mu \mathrm{m}$ deformation of $\mathrm{S}$-DEA, the charge quantity stored in the electrode was basically constant throughout this measurement like O-DEA shown in Fig. 6. As expected, after the observation of experimental results of O-DEA, S-DEA exhibited quite precise deformation response to the voltage application. It is strongly 


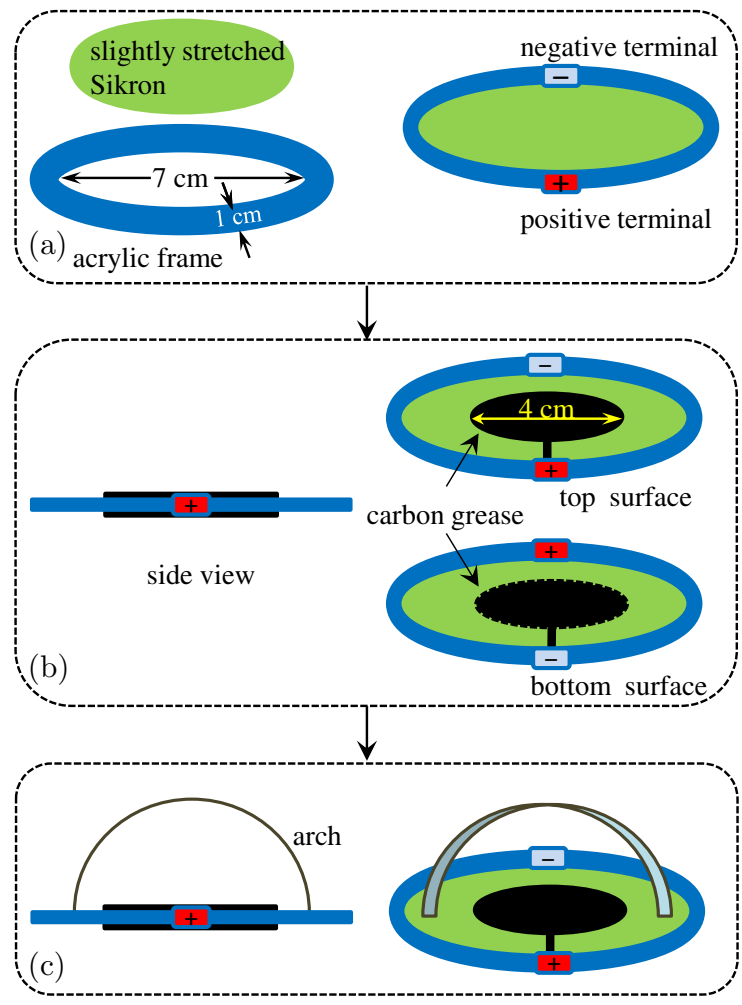

Fig. 7. Fabrication procedure of S-DEA.

speculated that the precisely controllable-shape change behavior is the characteristic DEAs commonly possess irrespective of the kinds of elastomer used.

\section{Conclusion}

Our study shows that even a simply structured DEA actually can exhibit an autonomous oscillatory shape change. Frequency of oscillation is fast for a soft actuator. Although this outcome is not a big leap toward the achievement of practical DEA, this is the first successful outcome of achieving the autonomous oscillatory shape change of DEA to our best knowledge. Furthermore, we observed that the DEA exhibits quantitatively quite precise deformation in response to the external electric stimulation, although the shape of most of electroactive polymers cannot be so precisely controlled.
Therefore, we strongly anticipate the great potential usefulness of DEA.

\section{Acknowledgment}

This study was carried out under the financial support of New Energy and Industrial Technology Development Organization, Research and Development of Innovative Robotic Components and its effective Manner of Utilization using Next Generation Functional Materials. We are quite grateful to Okura Industrial CO., Ltd. (Kagawa, Japan) for providing us with the free sample of Silkron.

\section{References}

${ }^{1}$ Y. Haraa and Y. Yamaguchi, Development of a paper actuator with PEDOT: PSS thin-films as an electrode, Actuators 3, 285 (2014).

${ }^{2}$ L. Ionov, Hydrogel-based actuators: Possibilities and limitations, Mater. Today 17, 494 (2014).

${ }^{3}$ H. Okuzaki and Y. Osada, Classification and research trend of soft actuators, J. Jpn Soc. Precision Eng. 80, 709 (2014).

${ }^{4}$ K. Kruusamäe, A. Punning, A. Aabloo and K. Asaka, Self-sensing ionic polymer actuators: A review, Actuators 4, 17 (2015).

${ }^{5} \mathrm{~S}$. Chiba and M. Waki, Basic characteristics of dielectric elastomer and their applications, J. Jpn. Soc. Precision Eng. 8, 713 (2014).

${ }^{6}$ X. Chen, T. Jiang, Y. Yao, L. Xu, Z. Zhao and Z. L. Wang, $A d v$. Funct. Mater. 26, 4906 (2016).

${ }^{7}$ W. Lai, Characteristics of dielectric elastomers and fabrication of dielectric elastomer actuators for artificial muscle applications, Master thesis, Iowa State University (2011).

${ }^{8}$ P. White, S. Latscha and M. Yim, Modeling of a dielectric elastomer bender actuator, Actuators 3, 245 (2014).

${ }^{9}$ Y. Onouchi, M. Sasaki and H. Tamagawa, Current-controlled Selemion bending in the controlled humidity environment, Sens. Actuators B: Chem. 135, 465 (2009).

${ }^{10}$ M. Sasaki, W. Lin, H. Tamagawa, S. Ito and K. Kikuchi, SelfSensing Control of Nafion-based ionic polymer-metal composite (IPMC) actuator in the extremely low humidity environment, Actuators 2, 74 (2013).

${ }^{11}$ H. Ngetha, M. Sasaki, H. Tamagawa, S. Ito and K. Ikeda, The study of dynamic characteristics of selemion CMV-based IPMC actuators in humidity-controlled environments, J. Jpn. Soc. Appl. Eletromag. Mech. 23, 742 (2015). 